Aim To assess the effect of thrombus aspiration on TIMI flow grade and in-hospital mortality in PPCI-treated STEMI patients Main outcome measures: Post-PPCI TIMI flow grade and in-hospital mortality.

Method Analysis of prospectively collected data of all STEMI patients undergoing primary PCI between March 2008 and June 2011 at a regional tertiary centre.

Results A total of 2567 patients (mean age $63.2 \pm 13.4$ years, $70.3 \%$ male) were included in this analysis. TA was performed in 1095 patients $(42.7 \%)$ (the thrombectomy group), whereas 1472 patients (57.3\%) underwent PPCI without TA (the non-thrombectomy group). Post PPCI TIMI flow grade 3 was achieved in $94.6 \%$ in the thrombectomy group compared to $88.7 \%$ in the non-thrombectomy group (adjusted OR; 2.0, 95\% CI 1.41 to $2.83, \mathrm{p}=0.0001$ ). Overall inhospital mortality was $4.5 \%(n=115)$. In a logistic regression model adjusted for many confounders, the use of thrombus aspiration was associated with a significant reduction in in-hospital mortality ( $2.7 \%$ vs $5.8 \%$, adjusted OR; $0.514,95 \%$ CI 0.29 to $0.93, p=0.027)$. Other independent predictors of in-hospital mortality in this model were advanced age, total ischaemic time, admission systolic blood pressure and heart rate, pre-procedural TIMI flow, admission haemoglobin and creatinine and multi-vessel coronary artery disease.

Conclusions In this large observational study of "real world" and unselected STEMI patients, manual thrombus aspiration during PPCI was associated with a significant reduction in in-hospital mortality and an increase in the rate of post-procedural TIMI flow grade 3 . These findings further confirm the benefits of thrombus aspiration in these patients.

\section{THROMBECTOMY AND PLATELET GLYCOPROTEIN IIB/IIIA BLOCKADE FOR STENT THROMBOSIS}

doi:10.1136/heartjnl-2012-301877b.35

M M Akhtar, * D A Jones, S Gallagher, R Weerackody, E Sammut, K S Rathod, A Jain, C Knight, A Mathur, A Wragg. Barts \& The Royal London Hospital, UK

Background Both mechanical thrombectomy and glycoprotein (GP) IIb/IIIa antagonists have been shown to improve clinical outcomes following primary PCI for ST segment elevation myocardial infarction. There is limited data describing the use of these technologies in the setting of stent thrombosis. We aimed to assess the combined approach of mechanical thrombectomy with adjunctive glycoprotein (GP) IIb/IIIa antagonists in patients presenting with acute stent thrombosis.

\section{Abstract 035 Table 1}

\begin{tabular}{lclll}
\hline & $\begin{array}{l}\text { No GPIlb/IIla or } \\
\text { thrombectomy } \\
\mathbf{N}=\mathbf{2 1}\end{array}$ & $\begin{array}{l}\text { No GPIlb/IIla or } \\
\text { thrombectomy } \\
\mathbf{N = 9 2}\end{array}$ & $\begin{array}{l}\text { No GPIIb/IIla or } \\
\text { thrombectomy } \\
\mathbf{N = 8 8}\end{array}$ & p Value \\
\hline Age & $55.25 \pm 30.0$ & $64.05 \pm 11.7$ & $62.72 \pm 12.6$ & 0.060 \\
Gender (female) & $7(35.0 \%)$ & $29(31.5 \%)$ & $22(25.0 \%)$ & 0.517 \\
Previous MI & $10(50.0 \%)$ & $54(58.7 \%)$ & $60(68.2 \%)$ & 0.215 \\
Previous CABG & $1(5.0 \%)$ & $7(7.6 \%)$ & $5(5.7 \%)$ & 0.837 \\
Hypercholesterolaemia & $7(35.0 \%)$ & $53(57.6 \%)$ & $50(56.8 \%)$ & 0.165 \\
Hypertension & $11(55.0 \%)$ & $53(57.6 \%)$ & $51(58.0 \%)$ & 0.971 \\
DM & $4(20.0 \%)$ & $25(27.2 \%)$ & $24(27.3 \%)$ & 0.786 \\
EGFR <60 & $4(20.0 \%)$ & $19(20.7 \%)$ & $21(23.9 \%)$ & 0.851 \\
MV disease & $6(30.0 \%)$ & $43(46.7 \%)$ & $36(40.9 \%)$ & 0.359 \\
Card Shock & $2(10.0 \%)$ & $6(6.5 \%)$ & $6(6.8 \%)$ & 0.855 \\
EF $<40$ & $11(55.0 \%)$ & $34(37.0 \%)$ & $23(26.1 \%)$ & 0.035 \\
Troponin (mean) & $1.65 \pm 2.98$ & $1.05 \pm 2.15$ & $2.44 \pm 4.43$ & 0.210 \\
Procedural Success & $17(85.0 \%)$ & $85(92.4 \%)$ & $85(96.6 \%)$ & 0.139 \\
\hline
\end{tabular}

Methods This was an observational cohort study of 3040 patients who underwent PPCI from 2003 to 2011. The primary outcome was major adverse cardiac events (all cause mortality, myocardial infarction, target vessel revascularisation and stroke). Follow-up was for a median of 3.0 years (IOR 1.2-4.6 years). Patients were split into 3 groups; those who underwent PCI using mechanical thrombectomy and GP IIb/IIIa inhibitors, those receiving either GPIIb/IIIa inhibitor or mechanical thrombectomy and those receiving neither.

Results 201 patients (6.6\%) of all STEMI patients presented with stent thrombosis (ST). Overall, GPIIb/IIIa inhibitors were used in $176(87.6 \%)$ patients. Mechanical thrombectomy was used in 94 (46.7\%) patients. 88 (44\%) patients received both GPIIb/IIA inhibitors and thrombectomy, $92(46 \%)$ received either GPIIb/IIIa inhibitor or thrombectomy and $21(10 \%)$ received neither therapy. Baseline characteristics of these 3 groups are shown in Abstract 035 table 1. Patients receiving both therapies tended to have more successful procedures and have better post-procedural left ventricular function. Kaplan-Meier estimates of long-term major adverse cardiac events showed the lowest rates of events for those patients receiving both therapies, followed by those receiving a single therapy, with the highest rates of MACE in those receiving neither therapy $(p<0.0001)$ (Abstract 035 figure 1). All individual component of MACE were lower in those receiving both therapies. Age-adjusted Cox analysis showed a decrease in the hazard of death for those receiving both therapies compared to those receiving neither (HR 0.17 (95\% CIs 0.06 to 0.54 ) and this was maintained with multiple adjustment (HR 0.11 (95\% CIs 0.02 to 0.85 )

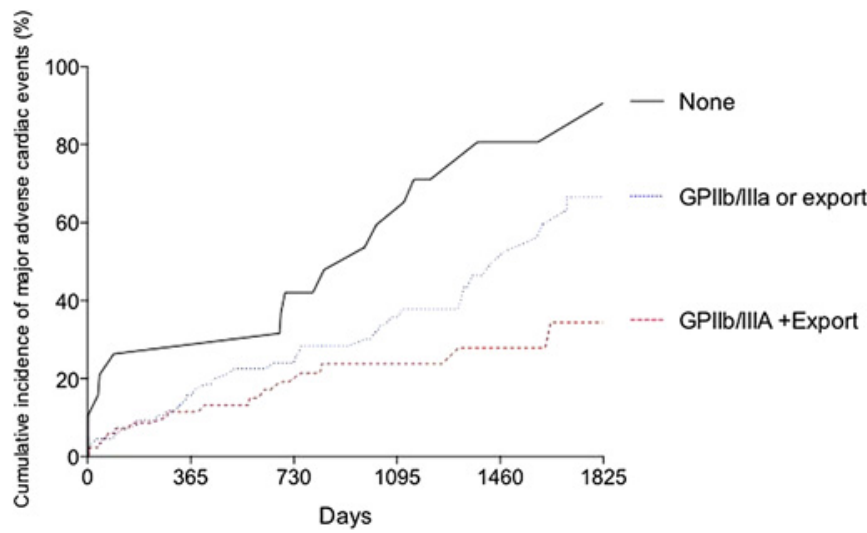

\section{Abstract 035 Figure 1}

Conclusion Prognosis after stent thrombosis is poor with high longterm event rates. Using a combined approach of mechanical thrombectomy with adjunctive GP IIb/IIIa blockade lead to effective primary PCI with improved long-term outcomes.

\section{NO DIFFERENCE IN LONG-TERM MAJOR ADVERSE CARDIAC EVENT RATES BETWEEN PACLITAXEL-ELUTING AND SIROLIMUS-ELUTING STENTS}

doi:10.1136/heartinl-2012-301877b.36

H A Abu-Own,* D A Jones, S M Gallagher, K S Rathod, A K Jain, C Knight, A Mathur, A Wragg. London Chest Hospital, UK

Background Previous studies have demonstrated similar outcomes over the short to mid term in patients treated with paclitaxeleluting stents (PESs) or sirolimus-eluting stents (SESs). However there is limited "real-world" data investigating long term outcomes. This study compared outcomes at 5 years following revascularisation in these two patient groups. 


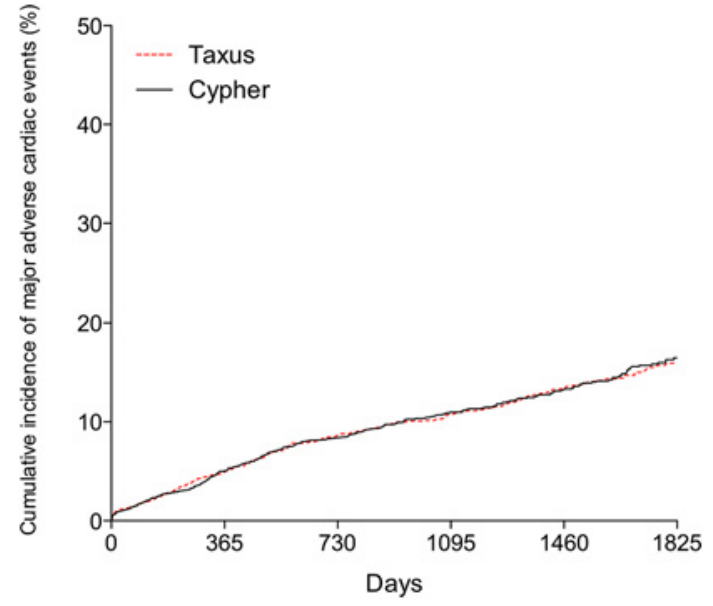

Abstract 036 Figure 1 MACE events over 5-year follow-up period.

Methods 4252 consecutive patients underwent PCI with either paclitaxel-eluting (PES) or sirolimus-eluting stents (SES) at a single centre (October 2003-January 2011). Indications for PCI included stable and unstable angina. Left main and vein graft lesions were excluded. Demographic and procedural data were collected at the time of intervention. All cause mortality data were obtained from the Office of National Statistics via the BCIS/CCAD national audit out to a median of 4.0 years ( $95 \%$ CI 2.4 to 5.6 years). Primary end point was major adverse cardiac events (MACE) a composite of all cause mortality, myocardial infarction and target vessel revascularisation (TVR).

Results There were 1592 (37\%) patients treated with SES and 2660 $(63 \%)$ patients treated with PES. Baseline demographic, angiographic, and procedural characteristics were similar between patients treated with PES and those treated with SES. At 5 years, there were no statistical differences in MACE between the stent types (SES $15.9 \%$ 95\% CI 12.7 to 19.4 vs PES: 16.5\% 95\% CI 12.6 to $20.3, \mathrm{p}=0.9)$. This consisted of similar rates of all-cause mortality ( $10.1 \%$ vs $9.3 \%, p=0.4)$, TVR (5.3\% vs $6.4 \%, p=0.4)$, and stent thrombosis ( $2 \%$ vs $1.8 \%, p=0.5)$. In diabetic patients $(n=1172$ $(28 \%))$, there was a trend towards lower MACE favouring PES but this did not reach statistical significance (19\% vs $24 \%, p=0.16$ ). On univariate and subsequent multivariate analysis there was no benefit from either stent type.

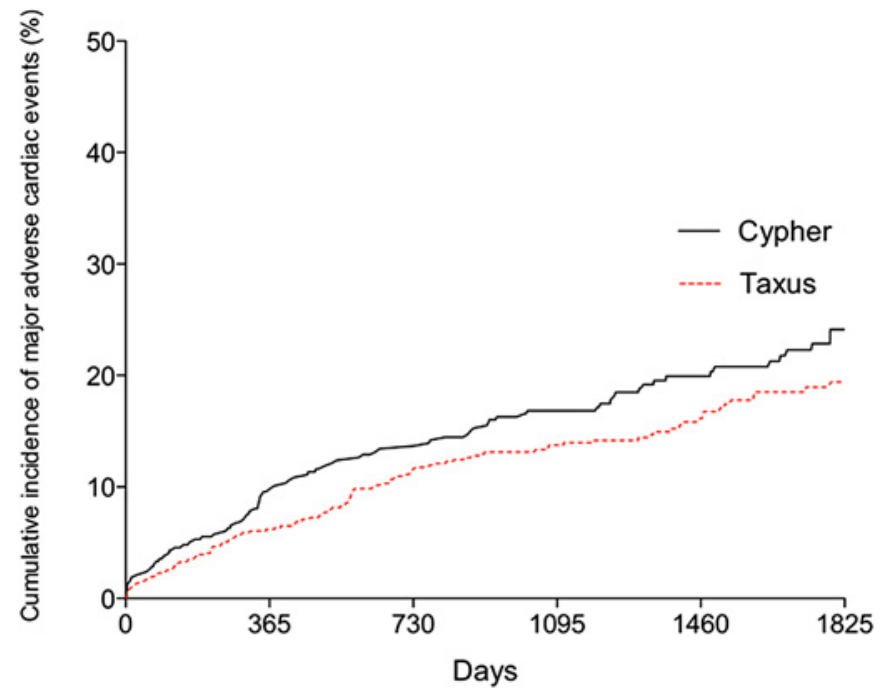

Abstract 036 Figure 2 MACE events in DM patients over 5-year followup period.
Conclusion This "real-world" observational analysis of DES-treated patients, PES and SES demonstrates similar overall safety and efficacy over a 5-year follow-up period with low rates of TVR.

\section{DRUG ELUTING STENTS (DES) OFFER BENEFIT OVER BARE METAL STENTS (BMS) INSERTED DURING VEIN GRAFT PCI}

doi:10.1136/heartjnl-2012-301877b.37

O P Guttmann,* D Jones, K Rathod, R Weerackody, A Jain, C Knight, A Mathur, A Wragg. Barts and The London NHS Trust, The London Chest Hospital, UK

Background Research trials have shown improved short-term outcome with DES over BMS in Saphenous vein graft (SVG) PCI, primarily by reducing target vessel revascularisation (TVR) for inStent Restenosis (ISR). Because of the high event rates in these patients it is not clear if these benefits are maintained long term. We compared the outcomes in patients undergoing SVG stent implantation treated with DES or BMS, over a prolonged follow-up period. Methods Clinical information was analysed from a prospective database on 512 patients who underwent PCI for SVG lesions between 2003 and 2010 at a London centre. Information was entered at the time of procedure and outcome assessed by all-cause mortality information provided by the Office of National Statistics via the BCIS/CCAD national audit. The primary end point used was major adverse cardiac events (MACE), defined as death, myocardial infarction (MI), stroke and target vessel revascularisation (TVR).

Results There were 293 patients who underwent PCI with BMS and 219 treated with DES. The mean follow-up period was $1086 \pm 418$ days for the BMS group and $1046 \pm 390$ days for the DES group. Baseline characteristics were the same between the groups other than diabetes, which was more common in the DES treated group $(24 \%$ vs $15 \%, p<0.0001)$. Per lesion there was a smaller average stent width used in the DES group $(3.2 \pm 0.05 \mathrm{~mm}$ vs $3.7 \pm 0.06 \mathrm{~mm}, \mathrm{p}=0.0001)$ however, there was no significant difference between the stent length $(20.1 \pm 0.60 \mathrm{~mm}$ vs $19.0 \pm 0.54 \mathrm{~mm}$, $\mathrm{p}=0.20)$ or number of stents used (1.66 vs $1.68, p=0.56)$. There was similar use of distal protection devices $(39.2 \%$ vs $48.3 \%, \mathrm{p}=0.08)$ and procedural success $(93.7 \%$ vs $93.0 \%, p=0.78)$ in the two groups. There was a significant difference in MACE between the two groups in favour of DES (30.2\% BMS vs $18.9 \%$ DES group [ $p=0.04$ ] over the 5 -year follow-up. MACE was driven by in-stent restenosis in both groups and the difference between groups was due to increased TVR

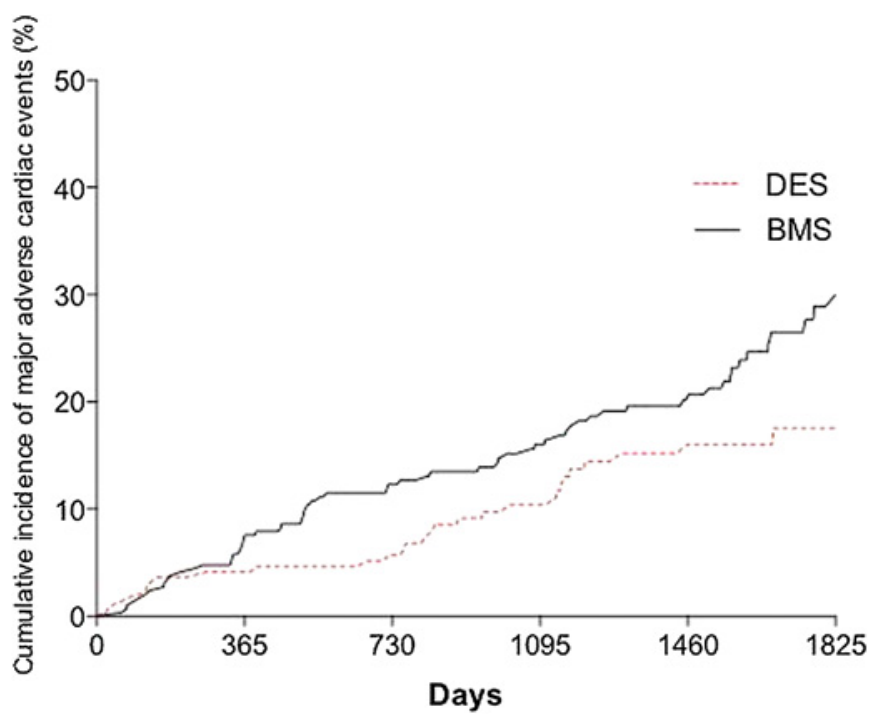

Abstract 037 Figure 1 MACE events over 5-year follow-up. 